\title{
Antibacterial activity and effects of Colla corii asini on Salmonella typhimurium invasion in vitro and in vivo
}

\author{
Kwang-il Park, Mi-ra Lee, Tae-woo Oh, Kwang-Youn Kim and Jin-yeul Ma*
}

\begin{abstract}
Background: Salmonella enterica serovar Typhimurium is a foodborne pathogen that triggers inflammatory responses in the intestines of humans and livestock. Colla corii asini is a traditional medicine used to treat gynecologic and chronic diseases in Korea and China. However, the antibacterial activity of Colla corii asini has been unknown. In this study, we investigated the antibacterial activity and effects of Colla corii asini extract on Salmonella typhimurium invasion.

Methods: To tested for antibacterial effects of Colla corii asini extracts, we confirmed the agar diffusion using Luria solid broth medium. Also, we determined the MIC (minimum inhibitory concentration) and the MBC (minimum bactericidal concentration) value of the Colla corii asini ethanol extract (CEE) by using two-fold serial dilution methods. We evaluated the expression of salmonella invasion proteins including SipA, SipB and SipC by using Western blot and qPCR at the concentration of CEE without inhibition of bacterial growth. In vitro and vivo, we determined the inhibitory effect of invasion of $S$. typhimurium on CEE by using gentamicin assay and $S$. typhimurium-infected mice.

Results: CEE significantly inhibited the growth of Salmonella typhimurium in an agar diffuse assay and had an MIC of 0 . $78 \mathrm{mg} / \mathrm{ml}$ and an MBC of $1.56 \mathrm{mg} / \mathrm{ml}$. Additionally, CEE reduced Salmonella typhimurium cell invasion via the inhibition of Salmonella typhimurium invasion proteins, such as SipA, SipB and SipC. Furthermore, CEE significantly suppressed invasion in the small intestines (ilea) of mice injected with Salmonella typhimurium.

Conclusion: These findings show that Colla corii asini exerts antibacterial activity and suppresses Salmonella typhimurium invasion in vitro and in vivo. Together, these findings demonstrate that Colla corii asini is a potentially useful therapeutic herbal medicine for treating salmonella-mediated diseases.
\end{abstract}

Keywords: Colla corii asini, Salmonella typhimurium, Antibacterial activity, Cell invasion, Salmonella invasion protein

\section{Background}

Colla corii asini (donkey-hide gelatin, E-Jiao) is a wellknown traditional Chinese medicine [1] and has been used for more than 2000 years in Asia for health-care food [2]. Previous reports have shown that the main components of Colla corii asini comprise amino acids, microelements and small molecular weight hydrolysates of collagen proteins [3]. Some pharmacological properties of Colla corii asini include sedation, anticoagulation, vasodilatation, hematopoiesis, as well as the improvement of cellular immunity and radio-protection $[3,4]$. It has been widely

\footnotetext{
* Correspondence: jyma@kiom.re.kr

Korean Medicine (KM)-Application Center, Korea Institute of Oriental Medicine (KIOM), 70 Cheomdan-ro, Dong-gu, Daegu 41062, Republic of Korea
}

(c) The Author(s). 2017 Open Access This article is distributed under the terms of the Creative Commons Attribution 4.0 International License (http://creativecommons.org/licenses/by/4.0/), which permits unrestricted use, distribution, and reproduction in any medium, provided you give appropriate credit to the original author(s) and the source, provide a link to the Creative Commons license, and indicate if changes were made. The Creative Commons Public Domain Dedication waiver (http://creativecommons.org/publicdomain/zero/1.0/) applies to the data made available in this article, unless otherwise stated. used for the treatment of gynecologic diseases (e.g., dysmenorrhea, menoxenia, metrorrhagia, abortion) [5] and some chronic diseases (e.g., anxiety, insomnia, apostaxis, hemoptysis, hematuria, hemafecia) [6]. Also, Colla corii asini has the anti-aging effect through enhancing antioxidant activity, scavenging free radicals, and modulating aging-related gene expression in aging mice model [7]. Furthermore, Colla corii asini promotes anemia and optimizes hemoglobin components in pregnant women with thalassemia [8]. Despite the increasing number of studies on the pharmacological effects of Colla corii asini, the antibacterial effect of Colla corii asini has not yet been investigated.

The gram-negative bacterium Salmonella enterica serovar Typhimurium ( $S$. typhimurium) is the main 
cause of gastroenteritis in humans and animals around the world, and it occurs via bacterial gastrointestinal infections [9]. These bacterial infections are caused by oral intake of contaminated food or water or by fecal contamination [10, 11]. Salmonella epidemics associated with contaminated food have been shown to result from insufficient hygiene conditions. $S$. typhimurium is classified as a non-typhoidal serovar, which can infect a broad range of animals and humans and can cause acute intestinal inflammation and diarrhea [12]. The systemic spread of $S$. typhimurium may cause severe disease in people with underlying infections or immunodeficiency disorders and can trigger an enormous economic loss in animal husbandry. $S$. typhimurium relies on two distinct groups of genes, Salmonella pathogenicity islands (SPI)1 and SPI-2, which play pivotal roles in the colonization of the host [13]. SPI-1, which encodes a type III secretion system (T3SS), secretes effectors such as SipA, SipB and SipC, which promote host invasion in the small intestinal tract via the formation of actin filaments at the bacterial entry site [14]. In contrast, Salmonella pathogenicity islands 2 (SPI-2) are required for proliferation inside the host cells [15].

In this study, we investigated the antibacterial effects of Colla corii asini by using $S$. typhimurium in vitro and in vivo. Furthermore, our findings suggest clues that may aid in understanding the antibacterial action of Colla corii asini and determining whether Colla corii asini may be a good candidate for treating bacterial diseases.

\section{Methods}

\section{Source of bacterial strain and culture condition}

The gram-negative bacterium S. typhimurium KCTC 1926 was obtained from the Korean Research Institute of Bioscience \& Biotechnology (KRIBB). For the experiment, bacteria were cultured at $37{ }^{\circ} \mathrm{C}$ with shaking in a $5 \mathrm{ml}$ Luria-Bertani (LB) broth medium, until 0.5-0.6 OD value at $600 \mathrm{~nm}$, pelleted at $5000 \mathrm{xg}$ for $10 \mathrm{~min}$, washed in PBS, and resuspended in $1 \mathrm{ml}$ Dulbecco Modified Eagle Medium (DMEM) completed media for cell infection $\left(1 \times 10^{8} / \mathrm{ml}\right)$.

\section{Cell culture}

Human colorectal cancer cells, caco-2 cells were maintained in DMEM with $1 \%$ antibiotics (penicillinstreptomycin), and 10\% fetal bovine serum (FBS) at $37^{\circ}$ $\mathrm{C}$ in $10 \% \mathrm{CO}_{2}$. Cells were seeded at $1 \times 10^{6}$ cells per well in 6-well tissue culture plates containing or not coverslips and maintained as differentiated monolayers for 21-28 days, changing the media every $2-3$ days [16].

\section{Preparation of Colla corii asini extract}

Colla corii asini were purchased from the Korea Medicinal Herbs Association (Yeongcheon, Korea). The identification of Colla corii asini was confirmed by Professor KiHwan Bae of the College of Pharmacy, Chungnam National University (Daejeon, Korea), and all voucher specimens were deposited in the herbal bank in the Korea Institute of Oriental Medicine (KIOM, Korea).

To prepare the Colla corii asini ethanol extract (CEE), Colla corii asini (50 g) was grounded to powder, then suspended in $70 \%$ ethanol $(300 \mathrm{ml})$ on shaking incubator (100 rpm) for $24 \mathrm{~h}$ at $40{ }^{\circ} \mathrm{C}$. The solution was filtered through a nylon net filter $(60 \mu \mathrm{m}$; Millipore Co., Denver, MA, USA), and then deposited overnight. The supernatant was lyophilized, and then the dried pellet (the yield, $1.66 \%$ ) was stored at $-20{ }^{\circ} \mathrm{C}$ until use. Also, to prepared the Colla corii asini water extract (CWE), Colla corii asini $(50 \mathrm{~g})$ were placed in $1000 \mathrm{~mL}$ distilled water and then extracted during $2 \mathrm{~h}$ of heating at $115{ }^{\circ} \mathrm{C}$ (Gyeongseo Extractor Cosmos-600, Inchon, Korea), and the solution was filtered using standard testing sieves $(140 \mu \mathrm{m})$ (Retsch, Haan, Germany). The supernatant was lyophilized, and then the dried pellet (the yield, $38.70 \%)$ was stored at $-20{ }^{\circ} \mathrm{C}$ until use.

\section{Amino acid content in Colla corii asini}

The amino acid composition of Colla corii asini were assayed with an L-8900 amino acid analyzer (Hitachi, Japan) after hydrolysis of $20 \mathrm{mg}$ sample with $0.02 \mathrm{~N} \mathrm{HCl}$ at $110{ }^{\circ} \mathrm{C}$ for $24 \mathrm{~h}$. The Aspartic acid (Asp), Threonine (Thr), Serine (Ser), Glutamic acid (Glu), Glycine (Gly), Alanine (Ala), Cysteine (Cys), Valine (Val), Methionine (Met), Isoleucine (Ile), Leucine (Leu), Tyrosine (Tyr), Phenylalanine (Phe), Lysine (Lys), Histidine (His), Arginine (Arg), Proline (Pro), Valine (Val), $\beta$-alanine and ethanol amine content were quantified by standard peak values.

\section{Agar well diffusion assay}

The extracts were tested for antibacterial effects by using agar diffusion on Luria agar [17]. The solid agar was punched with $7 \mathrm{~mm}$ diameter holes using the steriled tips. The agar plates included $1 \% \mathrm{~S}$. typhimurium and were then treated with $100 \mu \mathrm{l}$ of Colla corii asini extract. The concentrations of the extracts $(\mathrm{EtOH}$ and water) applied were $0.25-2 \mathrm{mg} / \mathrm{ml}$, and ampicillin $(5-40 \mu \mathrm{g})$ was used as a positive control. The solid agar was then incubated at $37{ }^{\circ} \mathrm{C}$ for $24 \mathrm{~h}$. After incubation, the clear zone associated with each concentration was measured.

\section{Determination of the minimum inhibitory concentration and minimum bactericidal concentration}

To determine the MIC (minimum inhibitory concentration) of the CEE, we detected the lowest concentration that did not demonstrate visible bacterial growth after $24 \mathrm{~h}$ of incubation at $37^{\circ} \mathrm{C}$. The MIC was determined by using two-fold serial dilutions of the extracts [18]. 
The MBC (minimum bactericidal concentration) value was determined by sub-culturing the test dilutions on a solid medium and incubating the cultures at $37{ }^{\circ} \mathrm{C}$ for $24 \mathrm{~h}$. The MBC value indicated the lowest dilution concentration at which there was no bacterial growth on the solid medium.

\section{Analysis of protein secretion}

$S$. typhimurium $\left(1 \times 10^{8}\right.$ cells $)$ was incubated in 6-well plates. CEE was treated to S. typhimurium for $1 \mathrm{~h}$, and then collected the DMEM media, and centrifuged, and the supernatants $(2 \mathrm{ml})$ were collected. The supernatants were filtered $(0.45-\mu \mathrm{m}$ pore size), and the proteins were precipitated with $100 \%$ trichloroacetic acid (TCA) by using high-speed centrifugation $(14,000 \times \mathrm{g}$ for $30 \mathrm{~min})$. The pellet was washed in cold acetone and resuspended in PBS. The solutions contained proteins and were mixed with Sodium Dodecyl Sulphate (SDS) sample buffer and boiled for $5 \mathrm{~min}$. Each sample was separated by SDS-10\% polyacrylamide gel electrophoresis (SDS-PAGE). The expression of SipC protein were analyzed by Western blot. Bacteria were lysed using ice-cold lysis buffer, and the protein concentration were determined by bicinchoninic acid (BCA) assay. Equal amounts of protein $(30 \mu \mathrm{g} / \mathrm{mL})$ were electrophoresed on 4-10\% SDS-acrylamide gels and transferred to nitrocellulose membranes using an electric transfer system. Blots were incubated for overnight at $4{ }^{\circ} \mathrm{C}$ with primary antibody against anti-SipC (1:100; ABIN335178). After three times washes with TBS-T, the membranes were incubated with HRP conjugated goat anti-mouse IgG (1:2000) for $1 \mathrm{~h}$ at room temperature. The signals were detected with a chemiluminescence reagent (Millipore Corporation, Billerica, USA), and analyzed with the ChemiDoc Touch Imaging System (Bio-Rad, Hercules). Protein bands were quantified by densitometry using Image J

\section{Quantitative real-time PCR}

$S$. typhimurium $\left(1 \times 10^{8}\right.$ cells $)$ was incubated in 6-well plates. CEE was treated to S. typhimurium for $1 \mathrm{~h}$ and mRNA was purified using TRIzol reagent and chloroform. Reverse transcription was conducted in $20 \mu \mathrm{l}$ reactions with $1 \mu \mathrm{g}$ of total RNA transformed into cDNA using AccuPower Cycle Script RT premix (Bioneer). The measurements of SipA, SipB and SipC mRNA and $16 \mathrm{~s}$ rRNA were conducted under the following conditions: 45 cycles of $95{ }^{\circ} \mathrm{C}$ for $10 \mathrm{~s}, 60{ }^{\circ} \mathrm{C}$ for $20 \mathrm{~s}$, and $72{ }^{\circ} \mathrm{C}$ for $30 \mathrm{~s}$ using a LightCycler $480 \mathrm{II}$ (Roche, Rotkreuz, SWI). The mRNA level in each sample was quantified on the basis of the threshold cycle $(\mathrm{Ct})$. The target genes were normalized relative to the reference gene $16 \mathrm{~S}$ rRNA [19].

\section{Cell viability}

Cell viability was determined by using a Cell Counting Kit-8 (CCK-8) according to the manufacturer's instructions by utilizing Dojindo highly water-soluble tetrazolium salt (Dojindo Molecular Technologies, Inc., Rockville, MD, USA). [2-(2-methoxy-4-nitrophenyl)-3-(4-nitrophenyl)-5-(2,4-disulfophenyl)-2H-tetrazolium, monosodium salt] (WST-8) was reduced by dehydrogenases in cells, to forming a yellow product (formazan) generated by the activity of dehydrogenases in cells in a manner directly proportional to the number of live cells. Briefly, Caco- 2 cells $\left(1 \times 10^{3}\right.$ cells) were plated on a 96-filter plate. After CEE treatments $(1.56-0.04875 \mathrm{mg} / \mathrm{ml})$ for $24 \mathrm{~h}, 10 \mu \mathrm{l}$ of the CCK- 8 solution was added to each well of the plate and incubated for $1 \mathrm{~h}$ at $37{ }^{\circ} \mathrm{C}$ with $5 \% \mathrm{CO} 2$. Absorbance was measured at $450 \mathrm{~nm}$ using a precision microplate reader (Molecular Devices, Sunnyvale, CA, USA).

\section{Selectivity index (SI)}

SI was calculated dividing the $\mathrm{IC}_{50}$ value by the $\mathrm{MIC}$ value for CEE $[20,21]$.

Table 1 Contents of Amino Acids in Colla corii asini

\begin{tabular}{lll}
\hline & EtOH extract & Water extract \\
\hline \multicolumn{1}{c}{ Amino acid } & $\mathrm{mg} / \mathrm{g}$ & \\
Glycine (Gly) & 8.639 & 0.208 \\
Alanine (Ala) & 2.527 & 0.062 \\
Phenylalanine (Phe) & 0.586 & 0.005 \\
Leucine (Leu) & 0.68 & 0.014 \\
Histidine (His) & 0.108 & 0.002 \\
Arginine (Arg) & 0.301 & 0.043 \\
Lysine (Lys) & 0.19 & 0.006 \\
Aspartic acid (Asp) & 1.511 & 0.052 \\
Glutamic acid (Glu) & 1.317 & 0.034 \\
B-amino isobutyric acid & 1.049 & 0.234 \\
Y-amino-n-butyric acid & 0.216 & $\mathrm{ND}$ \\
Serine (Ser) & 0.591 & 0.020 \\
Threonine (Thr) & 0.326 & 0.008 \\
Proline (Pro) & 1.621 & 0.030 \\
Tyrosine (Tyr) & 0.503 & $\mathrm{ND}$ \\
Valine (Val) & $\mathrm{ND}$ & 0.039 \\
B-alanine & 0.618 & 0.0847 \\
Ethanolamine & $\mathrm{ND}$ & 0.0356 \\
Carnosine & 0.193 & 0.1446 \\
Hydroxyproline & 0.685 & 0.0165 \\
Total free amino acid & 21.662 & 1.040 \\
H.Not Detect & & \\
\hline & &
\end{tabular}

*ND:Not Detected 


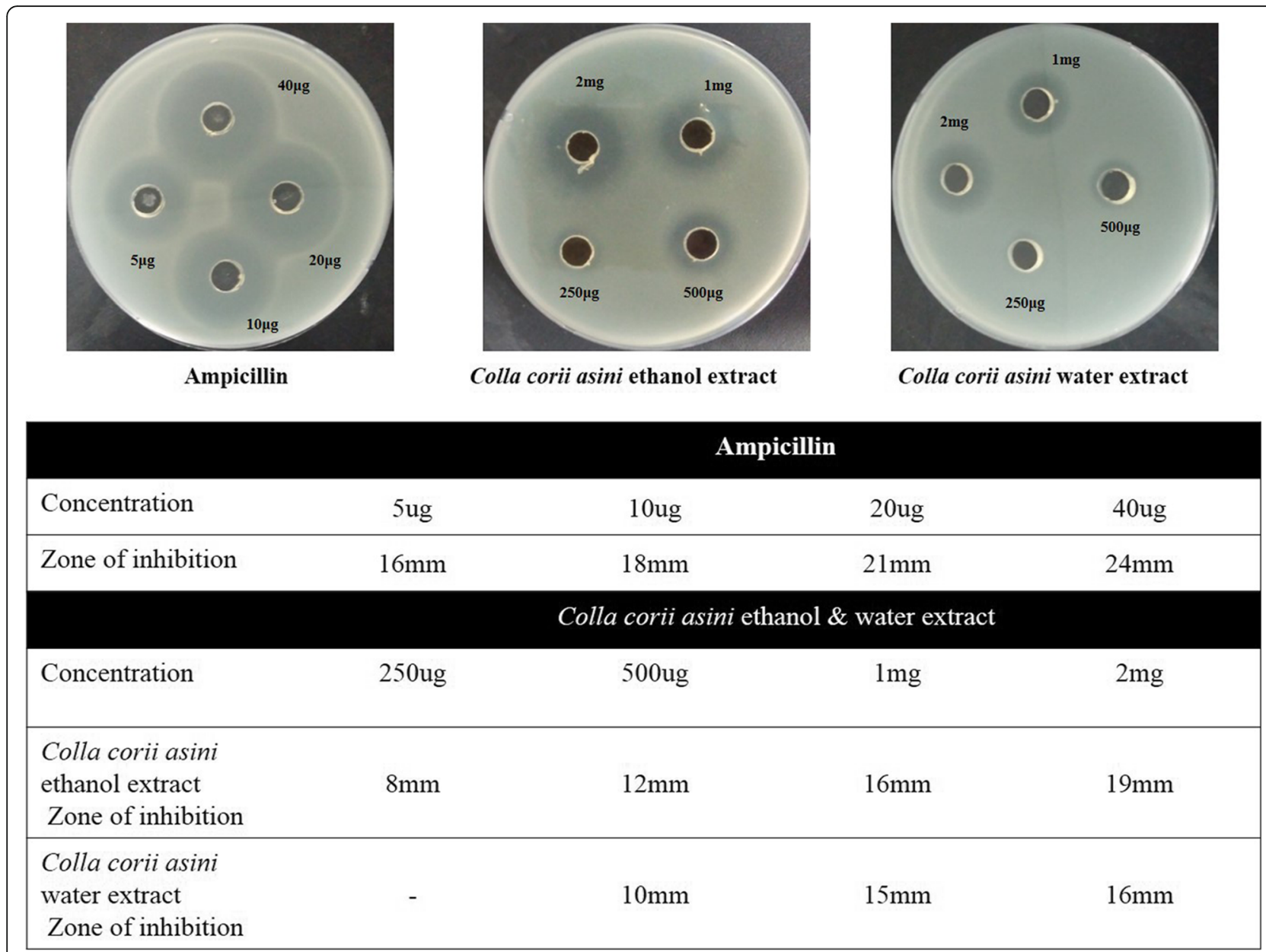

Fig. 1 The agar well diffusion assay of Colla corii asini extract. We determined the zone of inhibition for Colla corii asini extract and the positive control (ampicillin) after $24 \mathrm{~h}$. The zones of inhibition with the various concentrations of Colla corii asini ethanol and water extract were greater for S. typhimurium. The Colla corii asini ethanol extract had more of a significant effect than the Colla corii asini water extract

\section{Gentamicin assay}

$S$. typhimurium was used to infect Caco-2 cells $\left(1 \times 10^{6}\right.$ cell/well) in in 6 well plate. CEE was used to treat Caco2 cells with/without bacterial infection (50 multiplicity of infection (MOI)) in antibiotic-free medium, non- adherent bacteria were washed three times by PBS. Gentamicin $(100 \mu \mathrm{g} / \mathrm{ml})$ was then added for $1 \mathrm{~h}$ to kill any remaining bacteria. The Caco- 2 cells were washed three times with PBS and lysed with 1\% Triton-X-100. Bacteria in the lysis solutions were plated on solid agar,
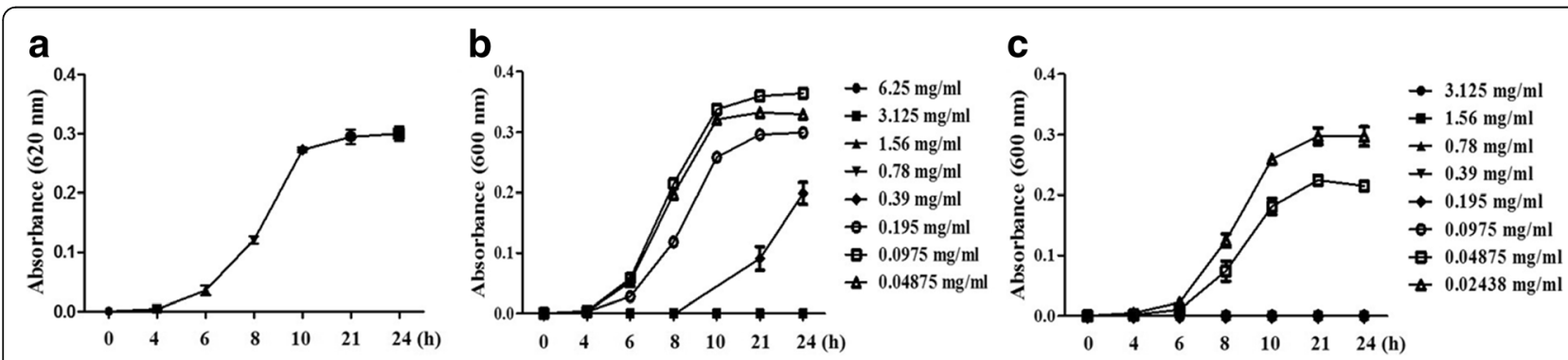

Fig. 2 The growth curves of S. typhimurium treated with Colla corii asini extract. We quantitatively evaluated the potency of the Colla corii asini ethanol extract by determining the MIC. a The growth curve of S. typhimurium with vechicle. $\mathbf{b}$ The growth curve of S. typhimurium with Colla corii asini ethanol extracts. c The growth curve of S. typhimurium with ampicillin. We calculated an MIC value of $0.78 \mathrm{mg} / \mathrm{ml}$ by using the tube dilution method. The growth of S. typhimurium was reduced by treatment with the Colla corii asini ethanol extracts. We calculated an MIC value of $0.78 \mathrm{mg} / \mathrm{ml}$ by using the tube dilution method 
and colony numbers were counted after $\mathrm{O} / \mathrm{N}$ incubation at $37^{\circ} \mathrm{C}[22]$.

\section{Infection of Salmonella enterica serovar Typhimurium in mice}

The infection of S. typhimurium was initiated in 6 to 7-week-old male ICR mice (Samtako Inc., Gyunggi-do, Korea). All mice were divided into 4 groups, each consisting of 5 mice. Mice were administered Colla corii asini extract (100 or $200 \mathrm{mg} / \mathrm{kg}$ ) via oral gavage daily. Mice were administered either $100 \mu \mathrm{l}$ of S. typhimurium $\left(5 \times 10^{7}\right.$ cells) or a vehicle control (PBS) by gavage. Forty-eight hours post-infection, mice were euthanized, and tissues (small intestine) were obtained from each treatment and the control group. Intestine samples were stored using the "Swiss roll" technique for immunohistochemistry (IHC) analysis [23]. All procedures were approved by the Korea Institute of Oriental Medicine Institutional Animal Care and Use Committee (KIOMIACUC) and were conducted in accordance with the US guidelines (NIH publication \#83-23, revised in 1985).

\section{Histological analysis}

After tissue fixation, Swiss rolled intestinal tissues were embedded in paraffin and were sectioned into $5 \mu \mathrm{m}$ sections using a microtome. The serial sections were subjected to immuno-fluorescence staining using fluorescence-tagged antibodies for measuring the number of $S$. typhimurium in the small intestine (ileum) using a confocal microscope.

\section{Statistical analysis}

For analysis of the statistical significance of differences between two groups we generally performed two-tailed Student's t-tests. For analysis of the statistical significance of differences among more than two groups, we performed one-way ANOVAs with Dunnett's multiple comparisons tests. All statistical analysis was performed using GraphPad Prism version 5.03 for Windows (GraphPad Software Inc., San Diego, CA, USA).

\section{Results}

\section{Content of amino acids in Colla corii asini}

Table 1 shows that CEE contained the major amino acids. Glycine $(8.639 \mathrm{mg} / \mathrm{g})$ has been the highest amino acid content in CEE. The total free amino acids content was $21.662 \mathrm{mg} / \mathrm{g}$.

\section{The antibacterial effect of Colla corii asini toward S. typhimurium}

To investigate the antibacterial effect of Colla corii asini, we performed an agar well diffusion assay. The negative control (vehicle) did not show bacterial growth around the well (data not shown). The antibacterial effect was determined on the basis of the zone of inhibition around
Colla corii asini extract and the positive control (ampicillin) after $24 \mathrm{~h}$ (Fig. 1). The zone of inhibition with the various concentrations of CEE and CWE increased in a dose-dependent manner for $S$. typhimurium. The CEE $(19 \mathrm{~mm})$ had more of a significant effect than the CWE (16 mm).

The antibacterial effect of the CEE and its potency were quantitatively evaluated by determining the MIC and MBC. In this study, the growth of $S$. typhimurium was significantly inhibited by the ethanol extract, thus resulting in an MIC of $0.78 \mathrm{mg} / \mathrm{ml}$, as determined by the tube dilution method (Fig. 2b), and the growth of $S$. typhimurium did not affect by vehicle (Fig. 2a). However, the growth of $S$. typhimurium with ampicillin inhibited at $0.0975 \mathrm{mg} / \mathrm{ml}$ (Fig. 2c). The MBC and MBC 50 values of the Colla corii asini ethanol extract was determined to be $1.56 \mathrm{mg} / \mathrm{ml}$ and $0.78 \mathrm{mg} / \mathrm{ml}$, respectively (Table 2). The results suggested that a high dose Colla corii asini can have an antibacterial effect.

CEE inhibits salmonella invasion proteins and mRNAs in $S$. typhimurium strains

$S$. typhimurium triggers intestinal diseases via the invasion of the small intestines. During this process, salmonella invasion proteins including $\mathrm{SipA}, \mathrm{SipB}$ and $\mathrm{SipC}$ play a critical role. To study the secretion of the effectors, we applied various concentrations of CEE to $S$. typhimurium $1 \mathrm{~h}$ before analysis. The MIC value was $0.78 \mathrm{mg} / \mathrm{ml}$; however, a value of $0.39 \mathrm{mg} / \mathrm{ml}$ slightly reduced bacterial growth. We determined that the maximum concentration was $0.195 \mathrm{mg} / \mathrm{ml}$ (no effect on bacterial growth) (Table 3). Proteins were obtained from culture supernatants and bacteria, and then analyzed by SDS-PAGE and Western blot to evaluate the secretion of T3SS-1 effectors in the bacterial supernatant (Fig. 3a). The expression of bands with sizes 80 (SipA), 68 (SipB) and 40 (SipC) $\mathrm{kDa}$ was evaluated for the inhibition of invasion proteins of $S$. typhimurium. The concentration of $0.195 \mathrm{mg} / \mathrm{ml}$ (lane 2) reduced the levels of salmonella invasion proteins such as SipA, SipB and SipC. Especially, SipC protein were significantly reduced by CEE on Western blot analysis. Additionally, we investigated the

Table $2 \mathrm{MBC}$ of Colla corii asini ethanol extract

\begin{tabular}{llllllll}
\hline $\begin{array}{l}\text { Colla corii asini } \\
\text { ethanol extract }\end{array}$ & $\begin{array}{l}\text { Dilution } \\
\text { rate }\end{array}$ & \multicolumn{1}{l}{ Colony number } & Average & Mean \\
\hline Control & $10^{7}$ & 300 & 285 & 121 & 143 & 212.2 & $4.5 \times$ \\
& $10^{8}$ & 58 & 45 & 98 & 73 & 68.5 & $10^{8}$ \\
$0.78 \mathrm{mg} / \mathrm{ml}$ & $10^{3}$ & 55 & 42 & 30 & 70 & 49.2 & $4.9 \times$ \\
& $10^{1}$ & 20 & 40 & 50 & 60 & 42 & 39 \\
$1.56 \mathrm{mg} / \mathrm{ml}$ & $10^{1}$ & 20 & 50 & 20 & 20 & 28 & \\
$3.12 \mathrm{mg} / \mathrm{ml}$ & $10^{1}$ & 50 & 50 & 60 & 30 & 48 & \\
$6.24 \mathrm{mg} / \mathrm{ml}$ & & & & &
\end{tabular}



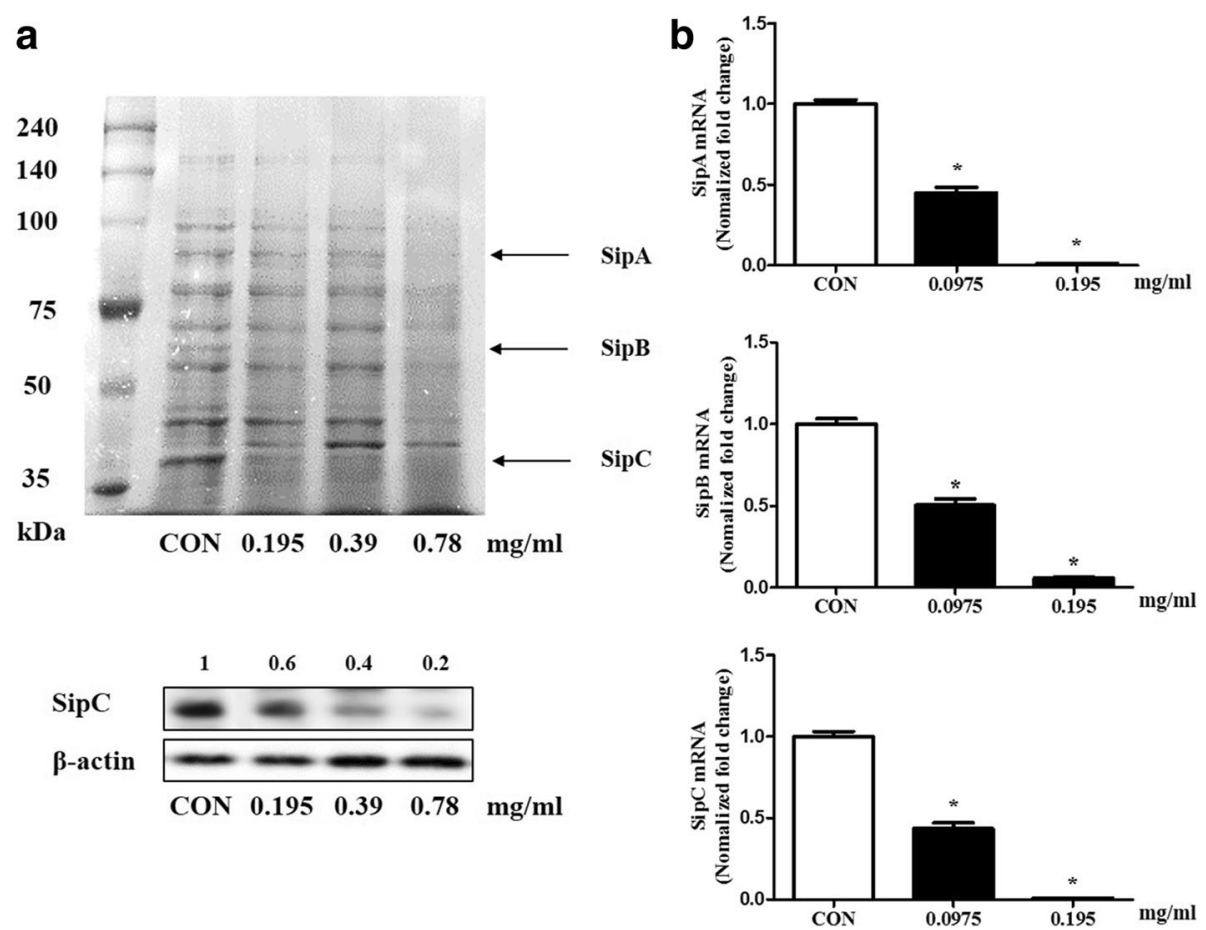

Fig. 3 Colla corii asini ethanol extract inhibited the proteins and mRNAs of T3SS-1 effectors in S. typhimurium. We detected the secretion of T3SS-1 effectors in the bacterial supernatant by western blotting. a The Colla corii asini ethanol extract $(0.195 \mathrm{mg} / \mathrm{ml})$ significantly reduced the salmonella invasion proteins such as SipA, SipB and SipC. b We treated S. typhimurium with various concentrations of Colla corii asini ethanol extract. After $24 \mathrm{~h}$, we extracted mRNA from S. typhimurium and conducted real-time PCR. Colla corii asini ethanol extract (0.195 mg/ml) significantly decreased the mRNA levels of salmonella invasion proteins

effects of Colla corii asini ethanol extract on mRNA levels of $S$. typhimurium invasion proteins by using realtime PCR analysis. $S$. typhimurium was treated with various concentrations of CEE for $1 \mathrm{~h}$ and mRNAs of invasion proteins, including SipA, SipB and SipC, were analyzed. The mRNA levels were significantly decreased at a $0.195 \mathrm{mg} / \mathrm{ml}$ concentration (Fig. 3b). The data showed that low-dose CEE had an antibacterial effect via the inhibition of the expression of invasion proteins.

Table 3 Determined the maximum concentration of Colla corii asini ethanol extract (no effect on bacterial growth)

\begin{tabular}{|c|c|c|c|c|c|c|c|}
\hline & $\begin{array}{l}\text { Dilution } \\
\text { rate }\end{array}$ & $\begin{array}{l}\text { Colc } \\
\text { num }\end{array}$ & $\begin{array}{l}\text { ny } \\
\text { iber }\end{array}$ & & Average & Total & Mean \\
\hline \multirow[t]{3}{*}{ Control } & $10^{7}$ & 19 & 85 & 50 & 52 & $\begin{array}{l}5 \times \\
10^{8}\end{array}$ & $\begin{array}{l}4.7 \times \\
10^{8}\end{array}$ \\
\hline & $10^{7}$ & 41 & 43 & 46 & 42 & $\begin{array}{l}4.2 \times \\
10^{8}\end{array}$ & \\
\hline & $10^{6}$ & 354 & 497 & 614 & 488.3 & $\begin{array}{l}4.9 \times \\
10^{8}\end{array}$ & \\
\hline \multirow{2}{*}{$\begin{array}{l}\text { Colla corii asini } \\
\text { ethanol extract } \\
(0.195 \mathrm{mg} / \mathrm{ml})\end{array}$} & $10^{7}$ & 47 & 56 & 60 & 54.3 & $\begin{array}{l}5.4 \times \\
10^{8}\end{array}$ & $\begin{array}{l}4.3 \times \\
10^{8}\end{array}$ \\
\hline & $10^{6}$ & 325 & 323 & 320 & 323.0 & $\begin{array}{l}3.2 \times \\
10^{8}\end{array}$ & \\
\hline
\end{tabular}

CEE inhibits the invasion of $S$. Typhimurium in Caco-2 cells

We investigated the effects of CEE on cell cytotoxicity in Caco- 2 cells. The Caco- 2 cell viability was measured after treatments with various concentrations of CEE (1.56-0.04875 mg/ml) and incubation for $1 \mathrm{~h}$. The CEE did not affect cell cytotoxicity (Fig. 4a). Also, we determined the $\mathrm{LC}_{50}$ value of Vero cells (derived from the kidney of an African green monkey) and calculated and presented in Table 4. Additionally, CEE significantly reduced the invasiveness of $S$. typhimurium in Caco-2 cells (Fig. 4b). The results suggested that low-dose Colla corii asini inhibited the invasion of the bacteria in vitro.

Table 4 Cytotoxicity ( $\mathrm{I}_{50}$ in $\mathrm{mg} / \mathrm{mL}$ ) of Colla corii asini ethanol extract and selectivity index (SI) values against Vero cells

\begin{tabular}{|c|c|c|c|}
\hline & $\begin{array}{l}\text { Antibacterial } \\
\text { activity } \\
\text { (MIC, mg/ml) }\end{array}$ & $\begin{array}{l}\text { Cytotoxicity } I C_{50} \\
(\mathrm{mg} / \mathrm{mL}) \\
\text { Vero Cells }\end{array}$ & $\begin{array}{l}\text { Selectivity } \\
\text { Index } \\
{ }^{I} C_{50} / \mathrm{MIC}\end{array}$ \\
\hline & $\begin{array}{l}\text { S. } \\
\text { typhimurium }\end{array}$ & & \\
\hline $\begin{array}{l}\text { Colla corii asini } \\
\text { ethanol extract }\end{array}$ & 0.78 & 4.75 & 6.09 \\
\hline
\end{tabular}



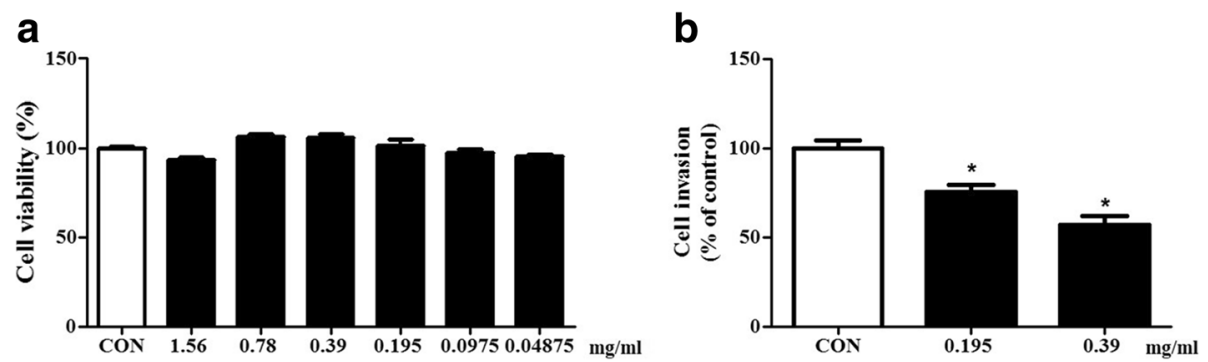

Fig. 4 The effect of Colla corii asini ethanol extract on cell invasion of S. typhimurium. $\boldsymbol{a}$ Colla corii asini ethanol extract did not have an effect on Caco-2 cell viability. $\mathbf{b}$ We treated S. typhimurium with various concentrations of Colla corii asini ethanol extract. After $1 \mathrm{~h}$, Caco-2 cells were washed three times with PBS, and gentamicin $(100 \mathrm{\mu g} / \mathrm{ml})$ was added for $1 \mathrm{~h}$ to kill any remaining bacteria. Caco-2 cell lysates were spread on solid agar. After $24 \mathrm{~h}$, we counted the colony numbers. Colla corii asini ethanol extract $(0.195 \mathrm{mg} / \mathrm{ml})$ significantly inhibited S. typhimurium invasion in Caco-2 cells

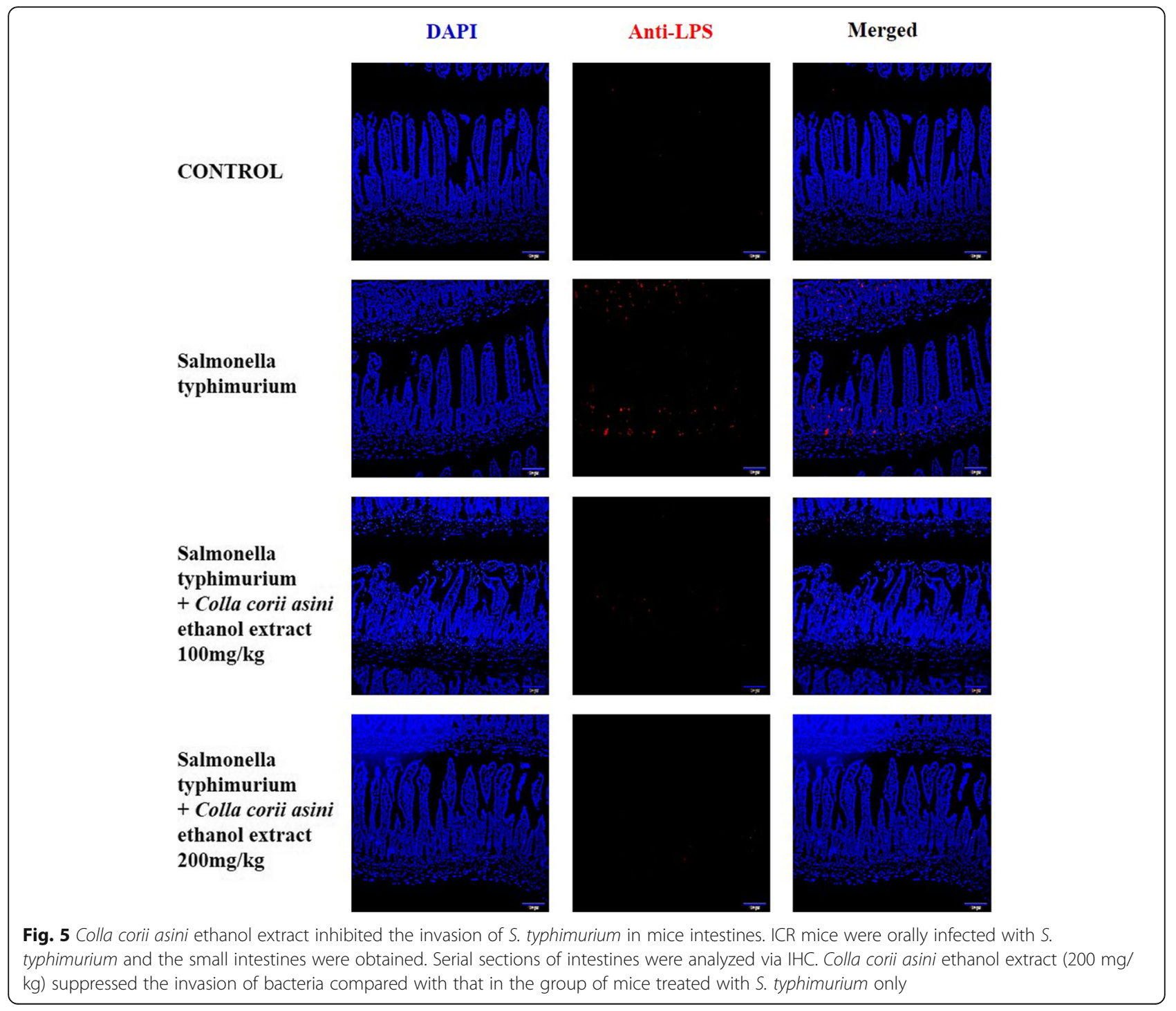




\section{CEE attenuated the invasion of $S$. Typhimurium in mice intestines}

To evaluate the function of CEE during a host infection by $S$. typhimurium, ICR mice were orally infected with $S$. typhimurium. The "Swiss rolled" tissues were divided into $5 \mu \mathrm{m}$ sections, and IHC was performed. The CEE attenuated the invasion of bacteria in a dose-dependent manner. Specifically, mice receiving the CEE treatment (200 $\mathrm{mg} / \mathrm{kg}$ ) had suppressed bacterial invasion, as compared with the group treated with $S$. typhimurium only (Fig. 5). The results suggested that low-dose Colla corii asini inhibited the invasion of bacteria in mice.

\section{Discussion}

$S$. typhimurium is the main cause of gastroenteritis, and it occurs via bacterial gastrointestinal infections from contaminated food, thus causing various diseases and enormous economic loss. Normally, $S$. typhimuriumrelated diseases occur when bacteria penetrate the gastro-intestines [24]. Despite the growing recognition of hygiene and health, salmonella infections have increased worldwide, and the underlying mechanisms remain unclear. Additionally, mutant bacteria and antibiotic resistance has increased, and new therapeutic agents without side effects are needed [25].

Colla corii asini (E'jiao) is prepared from the skin of Equus asinus and is used in traditional Chinese medicine and nutritional supplements in China and Korea [2]. We conducted experiments to determine the antibacterial effects of approximately four hundred kinds of Korean medicine on $S$. typhimurium, and Colla corii asini was the most effective. We hypothesized that Colla corii asini had an antibacterial effect through killing $S$. typhimurium as well as inhibiting the invasion of the host.

In the present work, the extracts obtained from Colla corii asini showed strong activity against $S$. typhimurium. Our data showed that CEE at a high dose (the inhibition of bacterial growth) possessed an antibacterial effect. An agar well diffusion assay and MIC/MBC values indicated that Colla corii asini might be the new antibacterial agent (Figs. 1 and 2, Table 2).

In previous studies, human and animal models of salmonella infection have investigated many TTSS effector proteins such as SipA, SipB and SipC and have shown that they are involved in inflammatory responses and diarrhea [26]. Salmonella invasion proteins play a pivotal role in the infection process and facilitate bacterial invasion in non-phagocytic cells in the host $[27,28]$. SipA is an important protein promoting salmonella invasion [29]. SipB is a substrate of the SPI1 type III export system [30] and deficient bacterial SipB do not enter eukaryotic cells [27]. SipC is a protein with diverse functions, and its actin nucleation and bundling activities play a critical role in salmonella-induced actin cytoskeleton reorganization and bacterial invasion [31]. Our data showed that CEE at low doses (no effect on bacterial growth) significantly decreased the expression of salmonella invasion proteins including SipA, SipB and SipC in $S$. typhimurium. Additionally, the mRNA expressions of salmonella invasion proteins were suppressed in a dose-dependent manner (Fig. 3a and b). To confirm the effect of CEE on salmonella invasion, we conducted gentamicin assays. CEE did not affect Caco-2 cell viability and attenuated the bacterial invasion in the Caco-2 cell monolayer (Fig. 4a and b). Furthermore, CEE inhibited the invasion of $S$. typhimurium in the small intestines (Fig. 5).

\section{Conclusions}

Our findings indicated that Colla corii asini contains potential antibacterial effects that may be a new pharmaceutical drug for treatment of $S$. typhimurium-related diseases. The ethanol extracts of Colla corii asini exert significant inhibitory effects against $S$. typhimurium via the inhibition of bacterial growth and cell invasion. The results of the study provide clues to understanding the antibacterial action of Colla corii asini.

\section{Abbreviations \\ CEE: Colla corii asini ethanol extract; MBC: Minimum Bactericidal Concentration; MIC: Minimum Inhibitory Concentration; S. typhimurium: Salmonella enterica serovar Typhimurium}

\section{Funding}

This work was supported by a grant (No. K17281, K17830) from the Korea Institute of Oriental Medicine funded by the Ministry of Education, Science and Technology (MEST), Republic of Korea. This work was also supported by Basic Science Research Program through the National Research Foundation of Korea (NRF) funded by the Ministry of Education (NRF2017R1D1A1B03032284)

Availability of data and materials

The datasets supporting the conclusions of this article are included within the article.

\section{Authors' contributions}

JYM developed the study design and revised the manuscript. KIP participated in the study design, analyzed the data, and wrote the draft manuscript. TWO and MRL carried out the experiments and analyzed the data. KYK was participated the revised the manuscript. All authors have read and approved the final manuscript.

\section{Ethics approval}

All procedures were approved by the Korea Institute of Oriental Medicine Institutional Animal Care and Use Committee (KIOM-IACUC) and were conducted in accordance with the US guidelines (NIH publication \#83-23, revised in 1985).

\section{Consent for publication}

Not applicable.

Competing interests

The authors declare that they have no competing interests.

\section{Publisher's Note}

Springer Nature remains neutral with regard to jurisdictional claims in published maps and institutional affiliations. 
Received: 3 April 2017 Accepted: 20 November 2017

Published online: 04 December 2017

\section{References}

1. ZR Gongheguo, wei sheng bu yao dian wei yuan hui: Pharmacopoeia of the People's Republic of China: Chemical Industry Press; 1997

2. Wu HZ, Yang F, Cui SY, Qin YF, Liu JW, Zhang YX. Hematopoietic effect of fractions from the enzyme-digested colla corii asini on mice with 5fluorouracil induced anemia. Am J Chinese Med. 2007:35(5):853-66.

3. Wu H, Ren C, Yang F, Qin Y, Zhang Y, Liu J. Extraction and identification of collagen-derived peptides with hematopoietic activity from Colla Corii Asini. J Ethnopharmacol. 2016;182:129-36.

4. Yao DF, Zhang YF, Zhou YF. Effects of Ejiao (colla corii asini) on the hemodynamics, hemorheology and microcirculation during endotoxin shock in dogs. Zhongguo Zhong yao za zhi. 1989;14(1):44-6. 64

5. Su N, Li B, Wang F, Quan S, Yang C, Shan D, Xing F. Clinical effectiveness of colla corii asini on improving uterine receptivity in controlled ovarian stimulation. J Trop Med. 2009;9:155-7.

6. Wang D, Ru W, Xu Y, Zhang J, He X, Fan G, Mao B, Zhou X, Qin Y. Chemical constituents and bioactivities of Colla corii asini. Drug discoveries \& therapeutics. 2014;8(5):201-7.

7. Wang D, Liu M, Cao J, Cheng Y, Zhuo C, Xu H, Tian S, Zhang Y, Zhang J, Wang F. Effect of Colla corii asini (E'jiao) on D-galactose induced aging mice. Biol Pharm Bull. 2012;35(12):2128-32.

8. Li Y, He H, Yang L, Li X, Li D, Luo S. Therapeutic effect of Colla corii asini on improving anemia and hemoglobin compositions in pregnant women with thalassemia. Int J Hematol. 2016;104(5):559-65.

9. Velge P, Wiedemann A, Rosselin M, Abed N, Boumart Z, Chausse AM, Grepinet O, Namdari F, Roche SM, Rossignol A, et al. Multiplicity of salmonella entry mechanisms, a new paradigm for salmonella pathogenesis. MicrobiologyOpen. 2012;1(3):243-58.

10. Sockett PN. The economic implications of human salmonella infection. The Journal of applied bacteriology. 1991;71(4):289-95.

11. Fang G, Araujo V, Guerrant RL. Enteric infections associated with exposure to animals or animal products. Infect Dis Clin N Am. 1991:5(3):681-701.

12. Schulte $M$, Hensel $M$. Models of intestinal infection by salmonella enterica: introduction of a new neonate mouse model. F1000Research. 2016:5

13. Hapfelmeier S, Stecher B, Barthel M, Kremer M, Muller AJ, Heikenwalder M, Stallmach T, Hensel M, Pfeffer K, Akira S, et al. The salmonella pathogenicity island (SPI)-2 and SPI-1 type III secretion systems allow salmonella serovar typhimurium to trigger colitis via MyD88-dependent and MyD88independent mechanisms. J Immunol. 2005;174(3):1675-85.

14. Galan JE, Curtiss R 3rd. Cloning and molecular characterization of genes whose products allow salmonella typhimurium to penetrate tissue culture cells. Proc Natl Acad Sci U S A. 1989:86(16):6383-7.

15. Shea JE, Beuzon CR, Gleeson C, Mundy R, Holden DW. Influence of the salmonella typhimurium pathogenicity island 2 type III secretion system on bacterial growth in the mouse. Infect Immun. 1999;67(1):213-9.

16. Yamashita S, Konishi K, Yamazaki Y, Taki Y, Sakane T, Sezaki H, Furuyama Y New and better protocols for a short-term Caco-2 cell culture system. J Pharm Sci. 2002;91(3):669-79.

17. Croze OA, Ferguson GP, Cates ME, Poon WC. Migration of chemotactic bacteria in soft agar: role of gel concentration. Biophys J. 2011;101(3): 525-34.

18. Holla G, Yeluri R, Munshi AK. Evaluation of minimum inhibitory and minimum bactericidal concentration of nano-silver base inorganic antimicrobial agent (Novaron((R))) against streptococcus mutans. Contemporary clinical dentistry. 2012;3(3):288-93

19. Mizusaki H, Takaya A, Yamamoto T, Aizawa S. Signal pathway in saltactivated expression of the salmonella pathogenicity island 1 type III secretion system in salmonella enterica serovar Typhimurium. J Bacteriol. 2008;190(13):4624-31.

20. Reyes-Melo K, Garcia A, Romo-Mancillas A, Garza-Gonzalez E, Rivas-Galindo VM, Miranda LD, Vargas-Villarreal J, Favela-Hernandez JMJ, MDR CamachoCorona. Meso-Dihydroguaiaretic acid derivatives with antibacterial and antimycobacterial activity. Bioorg Med Chem. 2017;25(20):5247-59.

21. Dzoyem JP, Melong R, Tsamo AT, Tchinda AT, Kapche DG, Ngadjui BT, McGaw L, Eloff JN. Cytotoxicity, antimicrobial and antioxidant activity of eight compounds isolated from Entada Abyssinica (Fabaceae). BMC research notes. 2017;10(1):118.
22. Kumar GA, Ramya V. Quantification of Gentamicin by microbial assay technique and reverse phase HPLC. European Journal of Experimental Biology. 2012;2(6):2083-9.

23. Ghaleb AM, McConnell BB, Kaestner KH, Yang WW. Altered intestinal epithelial homeostasis in mice with intestine-specific deletion of the Kruppel-like factor 4 gene. Dev Biol. 2011;349(2):310-20.

24. Majowicz SE, Musto J, Scallan E, Angulo FJ, Kirk M, O'Brien SJ, Jones TF, Fazil A, Hoekstra RM, International Collaboration on Enteric Disease 'Burden of Illness S. The global burden of nontyphoidal salmonella gastroenteritis. Clinical infectious diseases : an official publication of the Infectious Diseases Society of America. 2010;50(6):882-9.

25. Levy SB. The 2000 Garrod lecture. Factors impacting on the problem of antibiotic resistance. J Antimicrob Chemother. 2002:49(1):25-30.

26. Ehrbar K, Friebel A, Miller SI, Hardt WD. Role of the salmonella pathogenicity island 1 (SPI-1) protein InvB in type III secretion of SopE and SopE2, two salmonella effector proteins encoded outside of SPI-1. J Bacteriol. 2003; 185(23):6950-67.

27. Collazo CM, Galan JE. The invasion-associated type-III protein secretion system in salmonella-a review. Gene. 1997;192(1):51-9.

28. Pucciarelli MG, Garcia-del Portillo F. Protein-peptidoglycan interactions modulate the assembly of the needle complex in the salmonella invasionassociated type III secretion system. Mol Microbiol. 2003:48(2):573-85.

29. Jepson MA, Kenny B, Leard AD. Role of sipA in the early stages of salmonella typhimurium entry into epithelial cells. Cell Microbiol. 2001;3(6): $417-26$

30. Kaniga K, Trollinger D, Galan JE. Identification of two targets of the type III protein secretion system encoded by the inv and spa loci of salmonella typhimurium that have homology to the Shigella IpaD and IpaA proteins. J Bacteriol. 1995;177(24):7078-85.

31. Myeni SK, Zhou D. The C terminus of SipC binds and bundles F-actin to promote salmonella invasion. J Biol Chem. 2010:285(18):13357-63.

\section{Submit your next manuscript to BioMed Central and we will help you at every step:}

- We accept pre-submission inquiries

- Our selector tool helps you to find the most relevant journal

- We provide round the clock customer support

- Convenient online submission

- Thorough peer review

- Inclusion in PubMed and all major indexing services

- Maximum visibility for your research

Submit your manuscript at www.biomedcentral.com/submit
C) BioMed Central 